\title{
Research Progress of Rapid Rehabilitation Surgery in Anesthesia
}

\author{
FUZHAO XIANG, MING LI, FUQUAN. LUO, YUNMING YU, XING YANG, TAO. ZHANG AND YIN YANG* \\ Department of Anesthesiology, Three Gorges Hospital Affiliated to Chongqing University, Wanzhou 404000, China
}

\section{Yin Yang et al.: Rapid Rehabilitation Surgery in Anesthesia}

\begin{abstract}
Rapid rehabilitation surgery, also known as accelerated rehabilitation surgery, is a series of perioperative optimal management measures based on evidence-based medicine, in order to minimize the perioperative stress reaction of patients and reduce the risk of various complications, so as to achieve the goal of improving prognosis and promoting rapid recovery of patients. The concept of rapid rehabilitation surgery plays an important role in clinical anesthesia. It runs through the whole anesthetic work, such as preoperative anesthetic health education, preoperative fasting, selection of anesthetic scheme, intraoperative fluid therapy, intraoperative body temperature monitoring, postoperative analgesia and perioperative anesthetic management. The following article will review the application progress of rapid rehabilitation surgery in clinical anesthesia in recent years.
\end{abstract}

Key words: Rapid rehabilitation surgery, anesthesia, postoperative analgesia, fluid therapy, body temperature monitoring, perioperative anesthesia management

The concept of rapid rehabilitation surgery emphasizes clinical multidisciplinary cooperation, which was first put forward by Danish abdominal surgeon Henrik Kehlet in the 1990s. It is based on evidence based medicine to optimize perioperative management measures to reduce complications, improve prognosis and promote rapid postoperative recovery ${ }^{[1]}$. In recent years, with the continuous and rapid development of clinical medicine, the concept of rapid rehabilitation surgery is more and more widely used in clinic. Under the guidance of the concept of rapid rehabilitation surgery, not only anesthesiologists, surgeons and nurses are required to cooperate closely with each other, but also patients and their families need to cooperate closely with related work, so as to minimize the stress reaction caused by various medical measures during the perioperative period. So that the physiological function of patients can be balanced as far as possible, so as to speed up the rapid process of patients ${ }^{[2]}$. Anesthesia as an important part of the treatment of surgical patients, the concept of rapid rehabilitation surgery needs to run through the whole anesthetic work and plays an important role in many links of patients before, during and after operation. Therefore, this paper will review in detail the progress of the application of rapid rehabilitation surgery in clinical anesthesia in recent years, hoping to provide some guidance for the later clinical anesthesia. The concept of rapid rehabilitation surgery emphasizes the need for full communication with patients before operation, in order to further improve patients' cognitive level of surgical anesthesia and correct their misunderstandings of anesthesia. Susmita Bhattacharyya et al. ${ }^{[3]}$ reported that there is a close relationship between patients' cognitive level of anesthesia and their psychological status. The lack of knowledge about anesthesia is more likely to lead to a variety of bad emotions, such as anxiety, depression and fear. However, previous studies ${ }^{[4]}$ pointed out that further strengthening the health education of patients' knowledge related to anesthesia before operation can not only help them relieve their bad psychology before operation, but also further improve their satisfaction with clinical treatment. It plays a positive role in rapid recovery after operation. However, some scholars have pointed out that although preoperative anesthesia education can help patients improve their bad mood, it cannot effectively reduce the risk of postoperative adverse events, have no significant improvement on patients' pain and cannot effectively promote their functional recovery. But it can facilitate the development of postoperative analgesia. The application of the concept of rapid rehabilitation surgery has changed the traditional clinical concept of fasting before operation. In the past, for patients undergoing elective surgery, in 
order to prevent aspiration or reflux during surgery, patients are usually guided to fast before midnight, but there is no evidence based medical evidence that longterm preoperative eating can further improve the safety of patients and since 2009. The consensus guidelines for rapid rehabilitation surgery have pointed out that patients can eat an appropriate amount of solid food $6 \mathrm{~h}$ before anesthesia induction and drink an appropriate amount of transparent liquid $2 \mathrm{~h}$ before anesthesia induction $^{[5]}$. In addition, the European Society of Anesthesiology recommended in 2011 that patients should be allowed to eat an appropriate amount of solid food for $6 \mathrm{~h}$ and no water $2 \mathrm{~h}$ before surgery. Ji Wen $\mathrm{Z}$ et $a l .{ }^{[6]}$ reported that for patients who need surgical treatment, they can be instructed to eat an appropriate amount of carbohydrate rich liquid 2 to $3 \mathrm{~h}$ before operation and the intake can be controlled at about $300 \mathrm{ml}$, which can help patients improve preoperative anxiety and depression to a certain extent and help them to decompose protein after operation. In addition, it can also reduce postoperative insulin resistance. To prevent postoperative hyperglycemia caused by insulin resistance and increase the risk of postoperative incision infection. However, at present, oral carbohydrates before operation have not been generally recognized by most scholars., Russo M W et al. ${ }^{[7]}$ found that there was no significant difference in glucose metabolism and hemodynamics between patients who took carbohydrate drinks before operation and patients who took pure drinking water before operation and there was no difference in the incidence of postoperative complications between them. In the selection of clinical anesthesia, anesthesiologists used to focus on pain, hydrodynamic stability and vital signs, while the concept of rapid rehabilitation surgery advocated the use of combined anesthesia, compared with the traditional concept of anesthesia. It further increases the understanding of providing the best analgesia and how to minimize the surgical stress response of patients ${ }^{[8]}$. General anesthesia combined with local anesthesia is recommended for rapid rehabilitation surgery, especially for nerve block anesthesia and intraspinal anesthesia. It has been proved by a large number of studies that it can effectively reduce the stress response of patients in clinical anesthesia. It can improve the microcirculation and tissue perfusion of patients, can effectively reduce the inhibition of diaphragm activity and the analgesic effect is good. Gianni et al. reported that compared with general anesthesia, intraspinal anesthesia can reduce the rate of blood transfusion to a greater extent and reduce the incidence of postoperative complications, especially for patients with sleep apnea syndrome. Intraspinal anesthesia can effectively improve the safety of surgical treatment and reduce the risk of complications. In addition, under the concept of rapid rehabilitation surgery, anesthesia ${ }^{[9]}$ and analgesia management do not exist in isolation. The combination of anesthesia and analgesia management can effectively improve the effect of postoperative analgesia and reduce the dosage of opioids. In order to reduce the occurrence of drug related adverse reactions. In the previous study and analysis of the mortality of patients undergoing abdominal surgery, some scholars ${ }^{[10]}$ pointed out that compared with patients with single general anesthesia, the mortality rate of patients with general anesthesia combined with thoracic epidural analgesia should be reduced by at least $39.76 \%$. And the incidence of respiratory related or cardiovascular related complications is significantly lower and the recovery of gastrointestinal function is significantly faster after operation. The concept of rapid rehabilitation surgery emphasizes that the management of intraoperative fluid therapy should take the patient's physiological index as the end point and the principle of intraoperative fluid infusion should fully consider the optimal cardiac output of patients, especially for patients with lumbar anesthesia. In the case of ensuring the volume, we can use pressor drugs to stabilize the intraoperative mean arterial pressure, so as to ensure that the intraoperative blood supply of abdominal organs will not be affected to the greatest extent ${ }^{[11]}$. Jian W et al. ${ }^{[12]}$ carried out rapid rehabilitation surgical management on 100 patients who needed partial intestinal resection combined with anastomosis and divided them into group An and group B according to their different venous fluid management schemes. The former received reasonable fluid replacement according to their specific clinical manifestations, while the latter managed intraoperative intravenous fluid infusion according to the pulse volume monitored by esophageal Doppler ultrasound. The results showed that the total incidence of postoperative complications in group B was significantly lower than that in group $A(p<0.05)$. For surgical patients, the effect of intraoperative temperature maintenance will have a direct impact on their homeostasis. Mingdeng X et al. ${ }^{[13]}$ pointed out that patients with intraoperative body temperature below $36^{\circ}$ will significantly increase the risk of incision infection and the incidence of perioperative cardiac events after operation. In addition, in the recovery stage of patients, postoperative shivering will also increase 
their oxygen consumption to a certain extent. At the same time, some studies ${ }^{[14]}$ pointed out that patients with normal body temperature during operation had significantly less pain than patients with hypothermia during operation. However, at present, in the process of operation and anesthesia, the incidence of accidental hypothermia is still high, and related studies point out that its incidence ranges from $50 \%$ to $85 \%$. Therefore, it is particularly important to further strengthen the monitoring of patients' body temperature in the management of rapid rehabilitation surgery. It is particularly important to reduce the fluctuation of body temperature during operation. For surgical patients, perioperative pain is an unavoidable adverse reaction, mainly because a variety of perioperative medical procedures will inevitably cause some trauma to the patients. The local inflammatory reaction caused by traumatic tissue will stimulate the peripheral nerve to a certain extent ${ }^{[15]}$. The concept of rapid rehabilitation surgery can not only reduce the dosage of opioids to a certain extent, but also not affect the effect of postoperative analgesia by optimizing the perioperative pain management of patients. It can not only reduce the postoperative pain, but also promote the postoperative recovery ${ }^{[16]}$. In the past, opioid drugs were mainly selected in traditional clinical analgesia, although they have good analgesic effect, but they also have many side effects. It is easy to lead to respiratory depression and immune dysfunction and other complications ${ }^{[17]}$. Dickson E L et al.$^{[18]}$ reported that opioids can increase the risk of postoperative intestinal obstruction to some extent, so reducing the use of opioids can help to reduce the incidence of postoperative complications. At present, how to find an alternative opioid to reduce the use of opioids has become the first problem to be considered and solved in the pain management of rapid rehabilitation surgery. Some studies ${ }^{[19]}$ have pointed out that in postoperative analgesia in surgical patients, the use of acetaminophen and non-steroidal antiinflammatory drugs can reduce the use of opioids to some extent, but the effect of postoperative analgesia alone is not enough. It needs to be combined with multimode postoperative analgesia, such as local infiltration around the incision and epidural analgesia. In order to reduce the adverse reactions of patients after using local anesthetics, it is necessary to control the concentration of local anesthetics reasonably. Clonidine or opioids can be added to epidural analgesia to reduce the concentration of local anesthetics. However, it is necessary to guard against the possibility of hypotension in patients ${ }^{[20]}$. In recent years, continuous peripheral nerve block is more and more widely used in postoperative analgesia and its combination with adjuvants such as dexamethasone can further prolong the time of analgesia. However, it should be noted that adjuvants such as dexamethasone may lead to local vasoconstriction in patients ${ }^{[21]}$. Woojin $\mathrm{K}$ et al. ${ }^{[22]}$ reported that ice pack is simple, economical and has no side effects, so it has good application value in postoperative analgesia in patients undergoing abdominal surgery. At the same time, combined with oral antidepressants and anticonvulsants can not only reduce postoperative pain, but also reduce the use of opioids. Rapid rehabilitation surgery believes that postoperative nausea and vomiting is a common adverse reaction of patients, which will seriously increase patients' physical and mental discomfort. At the same time, a large amount of vomiting may also lead to reflux aspiration and water electrolyte imbalance, which is not conducive to wound healing and has a serious impact on postoperative recovery ${ }^{[23]}$. Therefore, it is necessary to strengthen the management of postoperative nausea and vomiting. Jan $\mathrm{S}$ et al. ${ }^{[24]}$ reported that general anesthesia can increase the probability of postoperative nausea and vomiting. Reducing the use of opioids can help patients control postoperative nausea and vomiting. If necessary, serotonin receptor antagonists and dexamethasone can be used. At the same time, some studies ${ }^{[25]}$ have confirmed that the use of corticosteroids can help patients reduce the incidence of postoperative vomiting. Getting out of bed as soon as possible after operation is also an important part of rapid rehabilitation surgery. If patients stay in bed for a long time after surgical treatment, not only increase the probability of complications such as thrombosis and pressure sores, but also reduce their pulmonary function and digestive function. Therefore, under the good management of analgesia, the physical conditions of patients need to mobilize patients as soon as possible, so that they can start rehabilitation exercise as soon as possible. In a word, as an important part of rapid rehabilitation surgery, the concept of clinical anesthesia has been gradually accepted by more and more anesthesiologists at home and abroad. As an innovative perioperative clinical pathway with multidisciplinary cooperation as the background, it runs through the whole perioperative period of patients and with the continuous development and optimization of the concept of rapid rehabilitation surgery and the increasing improvement of perioperative anesthesia management. Rapid rehabilitation surgery will play a more and more important role in ensuring 
the anesthetic effect and safety of patients and promoting their early recovery.

Author's contributions: Fuzhoao Xiang and Ming li contributed equally to this work

\section{Conflict of interests:}

The author declared no conflict of interests.

\section{REFERENCES}

1. Necib S, Tubach F, Peuch C, LeBihan E, Samain E, Mantz J, et al. PROMIFLUNIL trial group. Recovery from anesthesia after craniotomy for supratentorial tumors: comparison of propofol-remifentanil and sevoflurane-sufentanil. J Neurosurg Anesthesiol 2014;26:37-44.

2. El Saied MH, Mohamed NN, Mohamed HM, Amin MI. Dexmedetomidine versus fentanyl in anesthesia of cochlear implantation in pediatric patients. Egypt J Anaesth 2016;32 :55-9.

3. Bhattacharyya S, Tewari MK, Ghosh S, Chakroborty J, Das W, Barman U. Evaluation of the efficacy of bilateral sphenopalatine ganglion block in endoscopic sinus surgery under general anesthesia: a randomized prospective controlled trial. Res Opinion Anesth Intensive Care 2016;3:173.

4. Chaudhary O, Baribeau Y, Urits I, Sharkey A, Rashid R, Hess $\mathrm{P}$, et al. Use of Erector Spinae Plane Block in Thoracic Surgery Leads to Rapid Recovery From Anesthesia. Ann Thorac Surg 2020;110:1153-9.

5. Vergara-Amador E, Fuentes Losada LM, Moran Tobar AM. Rapid recovery anesthesia technique for ambulatory hand surgery. Salud Uninorte 2018;34:558-64.

6. Ji Wen Z, Anesthesia DO . Effect of Thoracic Paravertebral Nerve Block on the Rapid Recovery of Patients with Videoassisted Thoracic Surgery for Pulmonary Lobectomy. Systems Medicine 2018:65:10112-20.

7. Russo MW, Parks NL, Hamilton WG. Perioperative Pain Management and Anesthesia: A Critical Component to Rapid Recovery Total Joint Arthroplasty. Orthop Clin North Am 2017;48:401-5.

8. Lippell RM, Weinberg RY, Kelleher DC, Akerman MA, Tedore TR, White RS. Letter to the Editor on "Rapid Recovery after Total Joint Arthroplasty Using General Anesthesia". J Arthroplasty 2020;35:1444-5.

9. Biancofiore G, Tomescu DR, Mandell MS. Rapid recovery of liver transplantation recipients by implementation of fasttrack care steps: what is holding us Back? InSeminars in cardiothoracic and vascular anesthesia. Sage 2018;22:191-6.

10. Troup M, Zalucki OH, Kottler BD, Karunanithi S, Anggono V, van Swinderen B. Syntaxin1A neomorphic mutations promote rapid recovery from isoflurane anesthesia in Drosophila melanogaster. Anesthesiology 2019;131:555-68.

11. Shien LI, Anmin HU, Zhongjun Z. Clinical Application Progress of Non-Intubated Anesthesia under Spontaneous Breathing in Video-Assisted Thoracic Surgery. Med Recapitulate 2017;108:893-901.

12. Jian W, Na D, Xiaogang LI. Efficacy of intubated and nonintubated anesthesia in uniportal thoracoscopic surgery for thoracosympathectomy. J Shanxi Med University 2017;66:780-8.

13. Mingdeng X, Yuzhang A, Xiaoxiao X, Yucheng A, Xin W, Dianming J. Combined application of adductor canal block and local infiltration anesthesia in primary total knee arthroplasty: an updated meta-analysis of randomized controlled trials. Arch Orthop Trauma Surg 2021:1-4.

14. Iborra-Marcos A, Villanueva-Martinez $M$, Barrett SL, Sanz-Ruiz P. Ultrasound-Guided Decompression of the Intermetatarsal Nerve for Morton's Neuroma: A Novel Closed Surgical Technique. J Am Podiatr Med Assoc 2020;110:2332-40.

15. Novikov AY, Kovalev VA, Vinichuk NV, Chernyh YA, Golub IE, Sorokina LV. Prevention and correction of cognitive dysfunction after general anesthesia. Zh Nevrol Psikhiatr Im S S Korsakova 2017;117:28-31.

16. Borges J, Loureiro F. Minilifting technique for treatments of the lower third of the face and anterior cervical neck with tumescent anesthesia. J Cosmet Dermatol 2020;19:1208-10.

17. Garcia AL, Júnior MJ, Júnior EC, Noronha BR, dos Santos TI, Borges AH, et al. Surgical Treatment of Odontogenic Keratocyst with Application of Carnoy's Solution: Case Report. J Health Sci 2019;21:108-11.

18. Dickson EL, Stockwell E, Geller MA, Vogel RI, Mullany SA, Ghebre R, et al. Enhanced recovery program and length of stay after laparotomy on a gynecologic oncology service: a randomized controlled trial. Obstet Gynecol 2017;129:355-62.

19. Tsukamoto A, Niino N, Sakamoto M, Ohtani R, Inomata T. The validity of anesthetic protocols for the surgical procedure of castration in rats. Exp Anim 2018;67:329-36.

20. Kanaya A, Kuratani N, Nakata Y, Yamauchi M. Factors affecting extubation time following pediatric ambulatory surgery: An analysis using electronic anesthesia records from an academic university hospital. JA Clin Rep 2017;3:1-4.

21. Cappellini I, Picciafuochi F, Ostento D, Danti G, De Gaudio AR, Adembri C. Recovery of muscle function after deep neuromuscular block by means of diaphragm ultrasonography and adductor of pollicis acceleromyography with comparison of neostigmine vs. sugammadex as reversal drugs: study protocol for a randomized controlled trial. Trials 2018;19:1-7.

22. Kwon W, Bang S, Soh H, Jeong WJ, Lee SC, Choi BJ. Abdominal peripheral nerve block as the only anesthetic technique for totally extraperitoneal endoscopic inguinal hernia repair: Two case reports. Medicine 2018;97:10964-70.

23. Jiron JM, Mendieta Calle JL, Castillo EJ, Abraham AM, Messer JG, Malphurs WL, et al. Comparison of Isoflurane, Ketamine-Dexmedetomidine, and Ketamine-Xylazine for General Anesthesia during Oral Procedures in Rice Rats (Oryzomys palustris). J Am Assoc Lab Anim Sci 2019;58:40-9.

24. Jan S, Ali Z, Nisar Y, Naqash IA, Zahoor SA, Langoo SA, et al. A comparison of dexmedetomidine and clonidine in attenuating the hemodynamic responses at various surgical stages in patients undergoing elective transnasal transsphenoidal resection of pituitary tumors. Anesth Essay Research 2017;11:1079-83.

25. Ibrahim AI, Farag HM, Labib MM. The Effect of Anesthesia and Analgesia on Control of Breathing after Bariatric Surgery: A Systematic Review and Meta-Analysis of Randomized Control Trials. QJM: Int J Med 2020;113:hcaa039-6.

This is an open access article distributed under the terms of the Creative Commons Attribution-NonCommercial-ShareAlike 3.0 License, which allows others to remix, tweak, and build upon the work non-commercially, as long as the author is credited and the new creations are licensed under the identical terms

This article was originally published in a special issue,
"Clinical Research in Pharmaceutical and Biomedical
Sciences" Indian J Pharm Sci 2021:83(1)Spl issue1;116-119

